\title{
Optimisation du matériel de communication pour vaincre l'hésitation à la vaccination
}

\author{
Eve Dubé1,2*, Dominique Gagnon ${ }^{1}$, Maryline Vivion ${ }^{1}$
}

\section{Résumé}

L'hésitation à la vaccination (soit la réticence à accepter les vaccins recommandés) est un problème complexe qui complique la communication des risques pour les autorités sanitaires publiques et les cliniciens. Des études démontrent que fournir trop de données probantes sur l'innocuité et l'efficacité des vaccins aux personnes hésitantes à la vaccination ne permet pas de diminuer les croyances et craintes associées à l'hésitation. Le présent document a pour objectif de décrire les bonnes pratiques en matière d'élaboration de matériel de communication visant à contrer l'hésitation à la vaccination.

Nous avons évalué un échantillon de matériel de communication sur la vaccination utilisé au Canada en s'appuyant sur les recommandations d'un comité d'experts sur l'efficacité de la communication des risques pour les produits de santé du Conseil des académies canadiennes (2015). Plusieurs produits de communication actuels pourraient être améliorés si on se conformait aux pratiques d'excellence en matière de communication des risques fondées sur des données probantes. Nous avons désigné cinq pratiques d'excellence. Premièrement, déterminer le public cible et établir la confiance. Deuxièmement, fournir de l'information tant sur les risques que les avantages de la vaccination, puisque la plupart des gens souhaitent obtenir des renseignements nuancés. Troisièmement, présenter les faits avant de parler des mythes. Quatrièmement, utiliser des supports visuels. Cinquièmement, tester le matériel de communication avant le lancement.

Mettre ces pratiques d'excellence en œuvre avec les produits de communication actuels ou à venir aidera les vaccinateurs (dont les médecins, infirmiers praticiens, pharmaciens et professionnels de la santé publique) à créer du matériel de communication sensible aux moyens complexes que les personnes utilisent pour traiter et valoriser l'information et, par conséquent, plus susceptible d'optimiser l'adoption des vaccins au sein de leur collectivité.
Cette oeuvre est mise à la disposition selon les termes de la licence internationale Creative Commons Attribution 4.0

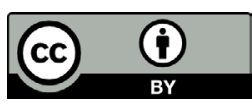

Affiliations

1 Institut national de santé publique du Québec, Québec, QC

${ }^{2}$ Centre de recherche du CHU de Québec-Université Laval, Québec, QC

${ }^{\star}$ Correspondance : eve.dube@inspq.qc.ca

Citation proposée : Dubé E, Gagnon D, Vivion M. Optimisation du matériel de communication pour vaincre I'hésitation à la vaccination. Relevé des maladies transmissible au Canada 2020:46(2/3):54-9.

https://doi.org/10.4745/ccdr.v46i23a05f

Mots-clés : hésitation à la vaccination, communication, adoption des vaccins, produits d'information, communication des risques, acceptation des vaccins, désinformation au sujet des vaccins

\section{Introduction}

Des études démontrent que fournir des données probantes sur l'innocuité et l'efficacité des vaccins aux personnes hésitantes à la vaccination a peu fait pour diminuer les croyances et craintes associées à l'hésitation. Des études antérieures démontrent que les messages qui donnent de l'information sur les vaccins et en font la promotion avec trop d'agressivité peuvent s'avérer contre-productifs avec les personnes qui hésitent déjà (1). Donner trop d'information peut même encourager I'hésitation (2). Pour aborder cet effet paradoxal de certains outils de communication, nous avons fait de la recherche pour connaître les techniques et stratégies de communication les plus efficaces. Fischhoff et ses collègues (3) ont compilé les conclusions de recherche sur ce qui fonctionne (ou pas) en communication des risques pour la santé. Le comité d'experts sur l'efficacité de la communication des risques pour les produits de santé du Conseil des académies canadiennes (4) les a adopté en tant que bonnes pratiques. Dans le but d'explorer la dont façon le matériel de communication canadien reflète ces pratiques d'excellence, nous avons évalué un échantillon de matériel de communication utilisé au Canada sur la vaccination (sites Web, fiches d'information, affiches, vidéos, etc.) à l'aide des bonnes pratiques de Fischhoff (3). 
Bien que la communication de l'information soit l'un des principaux outils à la disposition des vaccinateurs, il est peu probable que l'information seule change l'acceptation des vaccins de manière spectaculaire. En raison de la quantité de ressources humaines et financières investies dans l'élaboration et la diffusion du matériel de communication sur la vaccination, il est toutefois essentiel d'optimiser ces outils pour veiller à ce qu'ils fonctionnent selon les attentes. Le présent document a pour objectif de décrire les bonnes pratiques en matière d'élaboration de matériel de communication visant à vaincre I'hésitation à la vaccination $(5,6)$.

\section{Observations générales}

Notre analyse indique qu'on pourrait améliorer le matériel de communication actuel au Canada pour mieux correspondre aux pratiques d'excellence établies en matière de communication des risques. Nous constatons que la plupart des outils de communication sont axés sur les maladies pouvant être prévenues par un vaccin, les risques associés aux événements indésirables et la réfutation des mythes courants. En général, les démarches utilisées pour réfuter les mythes sont axées sur le mythe même plutôt que sur les bons renseignements. Nous constatons que l'information sur le risque est surtout qualitative (p. ex. « le risque d'événement indésirable après l'immunisation est faible " ou " les maladies qu'on peut éviter avec les vaccins peuvent provoquer une pneumonie, la surdité, des lésions cérébrales, des troubles cardiaques, la cécité et la paralysie chez les enfants qui ne sont pas protégés »). Peu des outils utilisés font usage de probabilités pour quantifier les risques. Lorsqu'il y a des probabilités, elles sont unidirectionnelles ( $p$. ex. présenter uniquement le risque de maladie ou le nombre de cas lors d'une éclosion) plutôt que bidirectionnelles (p. ex. présenter le risque de maladie et le risque d'événement indésirable après l'immunisation). Seule une minorité du matériel utilise des graphiques ou des vidéos.

Nous avons ensuite procédé à un balayage limité du matériel international, pour découvrir que certains outils de communication sont conformes à ces pratiques d'excellence et nous pourrions les adapter à l'intention des parents canadiens (p. ex. http://talkingaboutimmunisation.org.au/).

\section{Pratiques d'excellence}

Pour contrer l'hésitation à la vaccination, il faut des stratégies sur mesure mises à l'essai, fondées sur des données probantes et qui reconnaissent que l'hésitation à la vaccination est complexe et propre au contexte, en plus de varier selon le temps, le lieu et le type de vaccin (7).

\section{Déterminer le public cible et établir la confiance}

"Comprendre le point de vue des personnes visées par les services d'immunisation et leur mobilisation vis-à-vis la question 》, écrit Goldstein et ses collègues, " est tout aussi important que l'information que les experts souhaitent communiquer. » (8). La quantité, le contenu et le type d'information nécessaires pour convaincre une personne hésitante à la vaccination d'accepter les vaccins diffèrent beaucoup des renseignements fondamentaux dont une personne déjà favorable à la vaccination et qui a l'intention de se faire vacciner a besoin. Les études indiquent que les personnes hésitantes à la vaccination sont des " chercheurs d'information actifs » qui recherchent des renseignements "nuancés » présentant les avantages et inconvénients de la vaccination, et ce, pour faire un choix éclairé $(9,10)$. D'habitude, leurs besoins en information ne sont pas comblés par l'information habituelle des autorités sanitaires publiques, et ce, puisqu'en général, ces renseignements ne font pas référence à des études scientifiques et on les perçoit souvent comme axés sur les avantages des vaccins sans discuter de leurs risques potentiels (7). II faut des stratégies particulières pour les personnes qui sont fortement contre la vaccination. Ce n'est pas l'objet du présent document, mais nous en parlerons dans un résumé à venir du CANVax.

Un facteur clé qui influence le processus décisionnel en matière de vaccination est la confiance envers l'efficacité et l'innocuité des vaccins, le système qui les fournit (dont la fiabilité et la compétence des services de santé et des professionnels de la santé) et les motivations des décideurs politiques, qui décident quels sont les vaccins nécessaires, (où et quand) (11). De nombreuses études démontrent que l'hésitation à la vaccination ne découle pas du fait d'être mal informé, mais reflète plutôt un manque de confiance général envers les médecins, les sources publiques et les sociétés pharmaceutiques (12-14). Dans ce contexte, la crédibilité perçue des établissements qui fournissent les renseignements sur la vaccination est souvent plus importante que l'information même (15), ce qui met en lumière l'importance de la transparence et de l'honnêteté (16). Il est également essentiel de présenter à la fois les avantages et préjudices potentiels des vaccins. Des études d'autres pays indiquent qu'il s'agit d'une démarche prometteuse pour accroître l'acceptation des vaccins (17-19).

La recherche démontre que les personnes confrontées à de l'information qui contredit leurs valeurs peuvent se sentir menacées et réagir de manière défensive. Cette situation crée de la résistance, ce qui renforce leurs croyances initiales et réduit les chances de se livrer au comportement souhaité (c.-à-d. l'acceptation de la vaccination) (20). Cependant, on peut structurer les messages de façon à aborder les valeurs des patients et à favoriser la confiance (21). Par exemple, lorsqu'on a présenté la vaccination pour le virus du papillome humain en tant que vaccination pour prévenir le cancer, il y a eu moins 
de résistance que lorsqu'on la présentait comme moyen de prévention des infections transmissibles sexuellement (22).

\section{Fournir tant les risques que les avantages de la vaccination}

Fournir de l'information sur les risques et avantages de la vaccination n'est pas aussi simple qu'il y paraît (3). Lorsqu'ils élaborent du matériel de communication, les professionnels de la santé doivent se montrer sensibles aux moyens complexes que les personnes utilisent pour traiter et intégrer l'information. II ne faut pas présumer que «les chiffres parleront d'eux-mêmes ». La manière dont le message est élaboré est aussi importante que le contenu (23) : tandis que le contenu des outils doit être fondé sur les données probantes scientifiques accessibles, l'élaboration doit se fonder sur la communication des risques (24).

Les pratiques d'excellence qui découlent de cette revue de la littérature comprennent fournir des données sur les risques et avantages de la vaccination, de même que fournir des renseignements qualitatifs essentiels:

- Fournir la probabilité numérique des risques et avantages de la vaccination

Les outils doivent définir clairement tant les risques que les conséquences potentielles du fait de ne pas être vacciné (risques de maladies pouvant être prévenues par les vaccins), ainsi que les risques d'événement indésirable après la vaccination. Il faut le faire non seulement avec des mots, mais aussi avec des chiffres. II faut garder les dénominateurs constants (p. ex. un sur $10000 ; 25$ sur 10000 ) et utiliser des nombres entiers au lieu de fractions ou de décimales $(25,26)$.
- Fournir des renseignements qualitatifs essentiels

Le matériel ne doit pas seulement présenter de l'information numérique qualitative sur les risques et avantages de la vaccination, mais également fournir de l'information qualitative visant à présenter les données probantes qui appuient ces estimations. Il faut mettre l'accent sur les renseignements essentiels et la raison pour laquelle il est essentiel que les gens les comprennent dans le but de prendre leur décision en matière de vaccination. Par exemple, les personnes pourraient ne pas réaliser que leur décision de vaccination individuelle a des répercussions sur l'immunité collective ou les parents pourraient ne pas comprendre qu'ils ont le choix de reporter la vaccination.

\section{Présenter les faits d'abord, puis aborder les mythes}

Un des principaux objectifs de la plupart des outils de communication sur la vaccination est de " corriger » les idées fausses en matière de vaccination. Toutefois, il faut concevoir le matériel de communication avec soin, et ce, puisque les tentatives pour contrer un mythe pourraient plutôt le renforcer (20). Lors de l'élaboration du matériel de communication, nous devons mettre l'accent sur les faits et non sur les mythes. La technique commune de mettre les mythes sur la vaccination en tête d'affiche en grosses lettres ne constitue pas la meilleure stratégie, puisque les gens se souviendront des mythes et non des faits. À la place, il faut communiquer le fait principal en tête d'affiche, puis suivre avec une explication alternative. Lorsqu'un mythe est démonté, on crée un vide dans l'esprit de la personne. Pour être efficace, le matériel de communication doit ensuite combler ce vide (figure 1).

Figure 1 : Aborder les mythes en matière de vaccination



Source : Adapté de http://adelaidephn.com.au/assets/What_autism.pdf 


\section{Utiliser des aides visuelles}

Les aides visuelles telles que les infographies et les vidéos peuvent parfaire la compréhension de l'information complexe sur les risques. Les études démontrent que les aides visuelles peuvent aider les personnes à comprendre les risques pour la santé, en particulier celles dont les aptitudes en calcul sont faibles $(27,28)$. Les aides visuelles comprennent les vidéos, les images, les grilles d'icônes (c.-à-d. une image qui utilise une forme répétée un nombre précis de fois, en général 10, 100 ou 1,000, avec certaines des formes modifiées, d'habitude d'une couleur différente, pour représenter une proportion) ou infographies (c.-à-d. une combinaison d'images et de texte visant à résumer rapidement une grande quantité d'information). Les graphiques peuvent faciliter la compréhension des renseignements numériques et les pictogrammes constituent la meilleure stratégie pour communiquer à la fois le point principal (la signification) et le compte rendu textuel (l'information exacte) (figure 2).

Figure 2 : Une brève introduction à la sécurité des vaccins par Immunisation Canada



Source : Source: https://www.youtube.com/watch?v=oTECcvMGO8k

\section{Mettre la communication à l'essai avant le lancement}

Il est important de tester le matériel de communication avant le lancement pour s'assurer qu'il fonctionne comme prévu pour le public cible. Les résultats peuvent surprendre - une étude indique que l'information donnée en formats de fréquence (p. ex. un nourrisson sur 10 aura de la fièvre après un vaccin) est perçue comme plus risquée que la même information transmise en termes probabilistes (p. ex. $10 \%$ des nourrissons auront de la fièvre après un vaccin) (27). De plus, les études indiquent que jusqu'à un adulte sur deux ne possède pas les aptitudes nécessaires pour interpréter les probabilités et autres concepts mathématiques $(27,28)$.
- Utiliser du matériel de communication clair et facile à comprendre

II faut utiliser un langage simple, des phrases courtes et des sous-titres. On évite le langage dramatique et les commentaires désobligeants qui sèment la discorde. II importe que les chiffres utilisés soient faciles à comprendre (28).

- Éviter d'obtenir l'effet inverse

En ce qui concerne les personnes qui ont des idées toutes faites, les confronter à des contre-arguments peut entraîner le renforcement de leurs idées (29). Il est important de tester le matériel de communication, et ce, puisque même les efforts conçus avec soin pour influencer les personnes qui ont des croyances inexactes peuvent, en fait, consolider ces croyances (29).

\section{Conclusion}

II ne faut pas uniformiser les messages sur les risques, puisque les approches personnalisées sont les plus efficaces. La plupart des gens recherchent des renseignements équilibrés sur les vaccins lorsqu'ils décident de les accepter ou non. Les gens font confiance à leur professionnel de santé lorsqu'il leur affirme qu'il y a de la désinformation au sujet des vaccins. Les personnes avec des opinions solides contre la vaccination pourraient ne pas changer d'avis, peu importe le message ou la façon dont il est communiqué; par conséquent, des messages courts pourraient suffire. Pour aborder l'éventail des croyances qui contribuent à I'hésitation à la vaccination, il faut du matériel de communication sur mesure et ciblé vis-à-vis ces systèmes de connaissances différents et les besoins d'information et préférences uniques de collectivités particulières $(8,23)$. Les mises à jour sur ce sujet publiées seront sur le site Web de CANVax (5).

\section{Déclaration des auteurs}

E. D. - Conceptualisation, supervision, rédaction (première ébauche)

D. G. - Rédaction (révision et mise en forme)

M. V. - Conservation des données, analyse formelle, rédaction (révision et mise en forme)

\section{Conflit d'intérêts}

Le $D^{\text {re }}$ Dubé fait état de subventions de l'Agence de la santé publique du Canada, du ministère de la Santé et des Services sociaux du Québec, du Fonds de recherche en santé du Québec, des Instituts de recherche en santé du Canada, du Réseau canadien de recherche sur l'immunisation et du Conseil de recherches en sciences humaines du Canada. Le Dre Vivion mentionne des subventions de l'Association canadienne de santé publique et du Réseau canadien de recherche sur l'immunisation au cours de l'étude. 


\section{Financement}

L'analyse du matériel de communication a reçu l'appui du Réseau canadien de recherche sur l'immunisation en vertu de la subvention 385094. La création des résumés du CANVax est appuyée par le Fonds de partenariat d'immunisation de l'Agence de la santé publique du Canada.

\section{Références}

1. Nyhan B, Reifler J. Does correcting myths about the flu vaccine work? An experimental evaluation of the effects of corrective information. Vaccine 2015 Jan;33(3):459-64. DOI PubMed

2. Scherer LD, Shaffer VA, Patel N, Zikmund-Fisher BJ. Can the vaccine adverse event reporting system be used to increase vaccine acceptance and trust? Vaccine 2016 May;34(21):2424-9. DOl PubMed

3. Fischhoff B, Brewer N, Downs J, editors. Communicating Risks and Benefits: An Evidence-based User's Guide. Silver Springs (MD): US Department of Health and Human Services; 2011. https://www.fda.gov/media/81597/download

4. Council of Canadian Academies. The Expert Panel on the Effectiveness of Health Product Risk Communication. Health Product Risk Communication: Is the Message Getting Through? Ottawa (ON): CCA, 2015. https://cca-reports.ca/ reports/health-product-risk-communication-is-the-messagegetting-through/

5. CANVax. Centre canadien de ressources et d'échange sur les données probantes en vaccination. Ottawa (ON). https://www.canvax.ca/fr

6. MacDonald NE, Dubé E. Nouvelle ressource permettant de résumer les données sur la vaccination provenant du Centre canadien de ressources et d'échange sur les données probantes en vaccination (CANVax). Relevé des maladies transmissibles au Canada 2020;46(1):17-21. DOI

7. MacDonald NE; SAGE Working Group on Vaccine Hesitancy. Vaccine hesitancy: Definition, scope and determinants. Vaccine 2015 Aug;33(34):4161-4. DOI PubMed

8. Goldstein S, MacDonald NE, Guirguis S; SAGE Working Group on Vaccine Hesitancy. Health communication and vaccine hesitancy. Vaccine 2015 Aug;33(34):4212-4. DOI PubMed

9. Wheeler M, Buttenheim AM. Parental vaccine concerns, information source, and choice of alternative immunization schedules. Hum Vaccin Immunother 2013 Aug;9(8):1782-9. DOI PubMed

10. Sobo EJ, Huhn A, Sannwald A, Thurman L. Information curation among vaccine cautious parents: Web 2.0, Pinterest thinking, and pediatric vaccination choice. Med Anthropol 2016 Nov-Dec;35(6):529-46. DOI PubMed
11. Dubé E, Vivion M, Sauvageau C, Gagneur A, Gagnon R, Guay M. "Nature Does Things Well, Why Should We Interfere?": Vaccine Hesitancy Among Mothers (Canada). Qual Health Res 2016 Feb;26(3):411-25. DOI PubMed

12. Attwell K, Ward PR, Meyer SB, Rokkas PJ, Leask J. "Do-ityourself": vaccine rejection and complementary and alternative medicine (CAM). Soc Sci Med 2018 Jan;196:10614. DOI PubMed

13. Ward PR, Attwell K, Meyer SB, Rokkas PJ, Leask J. Risk, responsibility and negative responses: a qualitative study of parental trust in childhood vaccinations. J Risk Res 2017;21(9):1117-30. DOI

14. Attwell K, Leask J, Meyer SB, Rokkas P, Ward P. Vaccine Rejecting Parents' Engagement With Expert Systems That Inform Vaccination Programs. J Bioeth Inq 2017 Mar;14(1):65-76. DOI PubMed

15. Yaqub O, Castle-Clarke S, Sevdalis N, Chataway J. Attitudes to vaccination: a critical review. Soc Sci Med 2014 Jul;112:111. DOI PubMed

16. MacDonald NE, Smith J, Appleton M. Risk perception, risk management and safety assessment: what can governments do to increase public confidence in their vaccine system? Biologicals 2012 Sep;40(5):384-8. DOI PubMed

17. Haase N, Schmid P, Betsch C. Impact of disease risk on the narrative bias in vaccination risk perceptions. Psychol Health 2019 Sep; (epub ahead of print:1-20. DOI PubMed

18. Nan X, Madden K. HPV Vaccine Information in the Blogosphere: How Positive and Negative Blogs Influence Vaccine-Related Risk Perceptions, Attitudes, and Behavioral Intentions. Health Commun. Nov;27(8):829-36. DOI

19. Daley MF, Narwaney KJ, Shoup JA, Wagner NM, Glanz JM. Addressing Parents' Vaccine Concerns: A Randomized Trial of a Social Media Intervention. Am J Prev Med 2018 Jul;55(1):44-54. DOI PubMed

20. Cook J, Lewandowsky S. The Debunking Handbook. St. Lucia, Australia: University of Queensland, 2011. https:// skepticalscience.com/docs/Debunking_Handbook.pdf

21. Kahan DM. Social science. A risky science communication environment for vaccines. Science 2013 Oct;342(6154):53-4. DOl PubMed

22. Vorpahl MM, Yang JZ. Who Is to Blame? Framing HPV to Influence Vaccination Intentions among College Students. Health Commun 2018 May;33(5):620-7. DOI PubMed

23. Parrish-Sprowl J. Vaccine hesitancy communication: what counts as evidence. Vaccine 2018 Oct;36(44):6529-30. DOl PubMed

24. Thomson A, Vallée-Tourangeau G, Suggs LS. Strategies to increase vaccine acceptance and uptake: from behavioral insights to context-specific, culturally-appropriate, evidence-based communications and interventions. Vaccine 2018 Oct;36(44):6457-8. DOI PubMed 
25. Downs J, Fischhoff B. Qualitative Information. In: Fischhoff B, Brewer N, Downs J, editors. Communicating Risks and Benefits: An Evidence-based User's Guide Silver Springs: US Department of Health and Human Services; 2011

(Chapter 8). https://www.fda.gov/about-fda/reports/ communicating-risks-and-benefits-evidence-based-usersguide

26. Fagerlin A, Peters E. Quantitative Information. In: Fischhoff B, Brewer N, Downs J, editors. Communicating Risks and Benefits: An Evidence-based User's Guide. Silver Springs: US Department of Health and Human Services; 2011 (Chapter 7). https://www.fda.gov/about-fda/reports/ communicating-risks-and-benefits-evidence-based-usersguide
27. Peters E. Numeracy and the perception and communication of risk. Ann N Y Acad Sci 2008 Apr;1128:1-7. DOI PubMed

28. Peters E. Beyond comprehension: the role of numeracy in judgments and decisions. Curr Dir Psychol Sci 2012;21(1):315. DOI

29. Nyhan B, Reifler J, Richey S, Freed GL. Effective messages in vaccine promotion: a randomized trial. Pediatrics 2014 Apr;133(4):e835-42. DOl PubMed 